Discrete Comput Geom 38:605-614 (2007)

DOI: $10.1007 / \mathrm{s} 00454-007-1352-5$

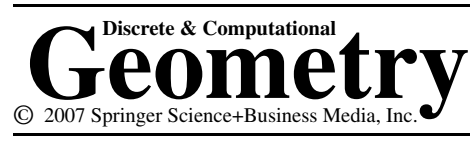

\title{
Concerning the Relationship between Realizations and Tight Spans of Finite Metrics*
}

\author{
Jack Koolen, ${ }^{1}$ Alice Lesser, ${ }^{2}$ and Vincent Moulton ${ }^{3}$ \\ ${ }^{1}$ Department of Mathematics, POSTECH, \\ Pohang, South Korea \\ koolen@postech.ac.kr \\ ${ }^{2}$ The Linnaeus Centre for Bioinformatics, Uppsala University, \\ Box 598, 75124 Uppsala, Sweden \\ alice.lesser@1cb.uu.se \\ ${ }^{3}$ School of Computing Sciences, University of East Anglia, \\ Norwich, Norfolk NR4 7TJ, England \\ vincent.moulton@cmp.uea.ac.uk
}

\begin{abstract}
Given a metric $d$ on a finite set $X$, a realization of $d$ is a weighted graph $G=\left(V, E, w: E \rightarrow \mathbb{R}_{>0}\right)$ with $X \subseteq V$ such that for all $x, y \in X$ the length of any shortest path in $G$ between $x$ and $y$ equals $d(x, y)$. In this paper we consider two special kinds of realizations, optimal realizations and hereditarily optimal realizations, and their relationship with the so-called tight span. In particular, we present an infinite family of metrics $\left\{d_{k}\right\}_{k \geq 1}$, and-using a new characterization for when the so-called underlying graph of a metric is an optimal realization that we also present-we prove that $d_{k}$ has (as a function of $k$ ) exponentially many optimal realizations with distinct degree sequences. We then show that this family of metrics provides counter-examples to a conjecture made by Dress in 1984 concerning the relationship between optimal realizations and the tight span, and a negative reply to a question posed by Althöfer in 1988 on the relationship between optimal and hereditarily optimal realizations.
\end{abstract}

\section{Introduction}

Let $(X, d)$ be a finite metric space, that is, a finite set $X$ together with a metric $d$ (i.e., a symmetric map $d: X \times X \rightarrow \mathbb{R}_{\geq 0}$ that vanishes precisely on the diagonal and that satisfies

* JHK was partially supported by the $\mathrm{Com}^{2} \mathrm{MaC}-\mathrm{SRC} / \mathrm{ERC}$ program of the MOST/KOSEF (Grant \# R111999-054) and also by a grant of POSTECH under Project Number 1RE0601301. JHK was also supported by KOSEF Grant \# NK0600501. VM was supported in part by EPSRC Grant EP/D068800/1. 
the triangle inequality). A realization of $d$ is a weighted graph $G=\left(V, E, w: E \rightarrow \mathbb{R}_{>0}\right)$ with $X \subseteq V$ such that for all $x, y \in X$ the length of any shortest path or geodesic in $G$ between $x$ and $y$ equals $d(x, y)$.

In this paper we consider two special kinds of metric realizations, which are defined as follows. For $G=(V, E, w)$ a weighted graph, let $\|G\|=\sum_{e \in E} w(e)$ denote the total edge weight of $G$.

- Optimal realizations: A realization $G$ of $d$ is called optimal if $\|G\|$ is minimal amongst all realizations of $d$. Note that optimal realizations always exist [5], [10], but they are not necessarily unique [5], [10], and in general it is NP-hard to find optimal realizations [1], [13].

- Hereditarily optimal, or h-optimal, realizations: These are defined inductively as follows. If $\# X \leq 2$, then any optimal realization of $d$ is defined to be h-optimal. If $\# X=k$ and h-optimal realizations have been defined for any metric on a set $Y$ with $\# Y<k$, then a realization $G=(V, E, w)$ of $d$ is defined to be h-optimal if $\|G\|$ is minimal with respect to the property that, for any $Y \subsetneq X$, there is some $E^{\prime} \subseteq E$ with $Y \subseteq V^{\prime}=\bigcup_{\{u, v\} \in E^{\prime}}\{u, v\}$ such that $\left(V^{\prime}, E^{\prime},\left.w\right|_{E^{\prime}}\right)$ is an h-optimal realization of $\left.d\right|_{Y}$. In [5] it is shown that h-optimal realizations always exist, and that, in contrast to optimal realizations, they are essentially unique (see [8] for more details).

In [5] some intriguing relationships between optimal realizations, h-optimal realizations and the so-called tight span of a metric are described, which we now recall. Let $P(d)$ be the polytope in $\mathbb{R}^{X}$ given by

$$
P(d)=\{f: X \rightarrow \mathbb{R}: f(x)+f(y) \geq d(x, y), \text { for all } x, y \in X\} .
$$

Then the tight span of $d$, denoted $T(d)$, is the polyhedral complex consisting of the bounded faces of $P(d)$ or, equivalently, those elements in $P(d)$ that are minimal with respect to the pointwise partial ordering given by $f \leq g$ if and only if $f(x) \leq g(x)$ for all $x$ in $X$ and $f, g \in P(d)$ [5]. Note that the map

$$
d_{\infty}: T(d) \times T(d) \rightarrow \mathbb{R}_{\geq 0} ; \quad d(f, g)=\max _{x \in X}|f(x)-g(x)|,
$$

is a metric on $T(d)$, and that $(X, d)$ can be embedded into $\left(T(d), d_{\infty}\right)$ via the map that takes each $x \in X$ to the element $h_{x} \in T(d)$ given by by putting $h_{x}(y)=d(x, y)$, for all $y \in X$ (see [9] for more details).

In Theorem 5 of [5], it is shown that if $G=(V, E, w)$ is an optimal realization of $d$, then there exists a map $\psi=\psi_{G}: V \rightarrow T(d)$ satisfying the conditions

(I) $\psi(x)=h_{x}$ for all $x \in X$, and

(II) $d_{\infty}(\psi(u), \psi(v))=w(\{u, v\})$ for all edges $\{u, v\} \in E$

(we call such maps proper). In addition, defining for $f \in T(d)$ the graph

$$
K(f)=\left(X,\left\{\{x, y\}: x, y \in\left(\begin{array}{l}
X \\
2
\end{array}\right) \text { and } f(x)+f(y)=d(x, y)\right\}\right),
$$

it follows as a consequence of Theorem 7 of [5] that the weighted graph $\Gamma_{d}=\left(V_{d}, E_{d}, w_{d}\right)$ with vertex set

$$
V_{d}=\{f \in T(d): K(f) \text { is connected and not bipartite }\},
$$



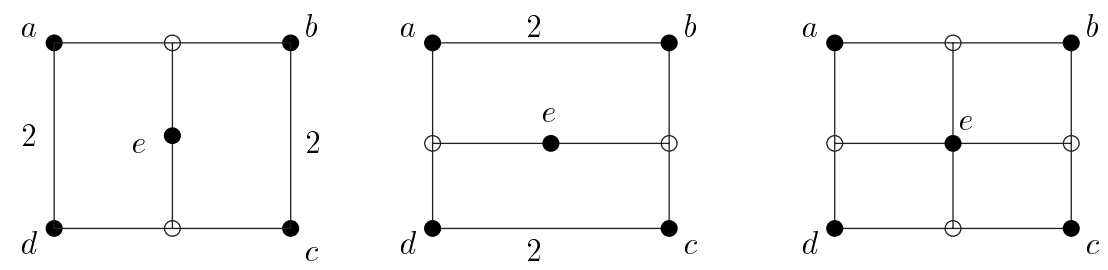

Fig. 1. Each graph realizes the same metric $D$ on the set $\{a, b, c, d, e\}$ (all unlabeled edges have weight 1). The realizations on the left and in the center are in fact optimal realizations of $D$, whereas the graph on the left is the h-optimal realization $\Gamma_{D}$ of $D$. Each of the optimal realizations can clearly be obtained by removing some subset of edges from the edge set of $\Gamma_{D}$.

edge set

$$
E_{d}=\left\{\{f, g\} \in\left(\begin{array}{c}
V_{d} \\
2
\end{array}\right): K\left(\frac{f+g}{2}\right) \text { is connected and bipartite }\right\}
$$

and weighting

$$
w_{d}: E_{d} \rightarrow \mathbb{R}_{>0} ; w_{d}(\{f, g\})=d_{\infty}(f, g)
$$

is an h-optimal realization of $d$ (see [8] for more details).

In [5, Remark (2), Section 3.2] Dress conjectured that for any optimal realization $G=(V, E, w)$ of $d$, any proper map $\psi: V \rightarrow T(d)$ is necessarily injective. Moreover, he conjectured that at least one and, in fact, all optimal realizations of $d$ can be obtained by deleting some subset of edges from the edge set of the h-optimal realization $\Gamma_{d}[5$, Section 3.20] (e.g., see Fig. 1). However, this latter conjecture was shown to be false by Althöfer [1], who showed that there are metrics having uncountably many optimal realizations, and who consequently posed the following related question [1, p. 117]: Let $G=(V, E)$ be a graph with $X \subseteq V$, and consider the set $W$ of all weight functions $w: E \rightarrow \mathbb{R}_{>0}$ for which $G=(V, E, w)$ is an optimal realization of $(X, d)$. Noting that $W$ is a compact convex polytope in $\mathbb{R}^{E}$, can the optimal realizations of $d$ corresponding to the extremals of $W$ be obtained by deleting some edges from the h-optimal realization of $d$ ?

In this paper we will show that Dress's conjecture concerning injectivity of proper maps is false, and that the answer to Althöfer's question is "no". We do this as follows. In Section 2 we recall some results concerning optimal realizations, and in Theorem 1 we prove an extension of Theorem 3.2 of [10], characterizing when the so-called underlying graph of a metric is an optimal realization of the metric. In Section 3 we present an infinite family of metrics $\left\{d_{k}\right\}_{k \geq 1}$, that generalize an example given by Imrich et al. in [10]. In particular, using Theorem 1 we find some optimal realizations of $d_{k}$ (Theorem 2), from which it follows that, for fixed $k$, the metric $d_{k}$ has (as a function of $k$ ) exponentially many optimal realizations with distinct degree sequences (Corollary 1). Finally, in Section 4, we show that the metric $d_{1}$ provides a negative answer to Althöfer's question, and that each metric $d_{k}, k \geq 2$, can be used to provide a counter-example to Dress's conjecture. 


\section{Optimal Realizations and the Underlying Graph}

Suppose that $(X, d)$ is a finite metric space. The underlying graph $U G(d)$ associated to $(X, d)[11]$ is the weighted graph with vertex set $X$, edge set consisting of those $\{x, y\} \in\left(\begin{array}{c}X \\ 2\end{array}\right)$, for which $d(x, z)+d(z, y)>d(x, y)$ for all $z \in X$ distinct from $x, y$, and edge-weighting defined by giving each edge $\{x, y\}$ weight $d(x, y)$. It is straightforward to check that $U G(d)$ is a realization of $d$. In this section we give a characterization for when $U G(d)$ is actually an optimal realization of $d$.

We begin by recalling two well-known results concerning realizations.

Lemma 1 [10, Lemma 3.1.i]. Suppose that $(X, d)$ is a finite metric space, and that $x, y, z \in X$ are distinct with

$$
d(x, y)+d(y, z)=d(x, z) .
$$

Suppose also that $\gamma_{x y}$ and $\gamma_{y z}$ are geodesics between $x, y$ and $y, z$, respectively, in some realization of $d$. Then the only vertex that $\gamma_{x y}$ and $\gamma_{y z}$ have in common is $y$.

Lemma 2 [10, Lemma 3.1.ii]. Suppose that $(X, d)$ is a finite metric space, and that $x, y, u, v \in X$ are distinct with

$$
d(x, y)+d(u, v)<\max \{d(x, u)+d(y, v), d(x, v)+d(y, u)\} .
$$

Then in any realization of $d$ every geodesic between $x$ and $y$ is disjoint from every geodesic between $u$ and $v$.

A well-known necessary condition for a realization $G=(V, E, w)$ of $d$ to be optimal is that for every edge $e \in E$ there is some pair $x, y \in X$ so that every geodesic between $x$ and $y$ in $G$ contains $e$ [1], [12]. We now sharpen this observation.

Lemma 3. Suppose that $(X, d)$ is a finite metric space, and that $G=(V, E, w)$ is an optimal realization of $d$. Then for all $e \in E$ there is some edge $\{x, y\}$ of $U G(d)$ for which every geodesic between $x$ and $y$ in $G$ contains $e$.

Proof. Suppose $e \in E$. Then by the result stated just before the lemma there is some pair $x, y \in X$ so that every geodesic between $x$ and $y$ in $G$ contains $e$. Let $x, y$ be such a pair with $d(x, y)$ minimal. We claim that $\{x, y\}$ is an edge in $U G(d)$.

Suppose that this is not the case. Then, since $\{x, y\}$ is not an edge of $U G(d)$, there must exist some $z \in X$ distinct from $x$ and $y$ with $d(x, z)+d(z, y)=d(x, y)$. In particular, it follows that there must be a geodesic $\gamma$ in $G$ between $x$ and $y$ containing $z$ and $e$. Without loss of generality, assume that $e$ is contained in the portion of $\gamma$ between $z$ and $y$. Then every geodesic in $G$ between $z$ and $y$ must contain $e$ (otherwise, using Lemma 1, we could find a geodesic in $G$ between $x$ and $y$ not containing $e$ ). However, $d(z, y)<d(x, y)$, which contradicts the choice of $\{x, y\}$.

We now give necessary and sufficient conditions for the underlying graph of a metric $d$ to be an optimal realization of $d$, in particular extending Theorem 3.2 of [10]. For 
$G=(V, E, w)$ a weighted graph, let $d_{G}$ denote the graph metric on the vertex set of $G$, defined by letting $d_{G}(u, v)$ equal the length of any geodesic in $G$ between $u$ and $v$, for all $u, v \in V$.

Theorem 1. Suppose that $(X, d)$ is a finite metric space. Then $U G(d)$ is an optimal realization of $d$ if and only if the following two conditions hold:

(i) For every pair of edges $\{x, y\},\{y, z\}$ in $U G(d)$ with $x, z$ distinct, $d(x, z)=$ $d(x, y)+d(y, z)$.

(ii) For every disjoint pair of edges $\{x, y\},\{u, v\}$ in $U G(d)$, eitherd $(x, y)+d(u, v)<$ $\max \{d(x, u)+d(y, v), d(x, v)+d(y, u)\}$ or $d(x, y)+d(u, v)=d(x, u)+$ $d(y, v)=d(x, v)+d(y, u)$.

Proof. We first prove the necessity of conditions (i) and (ii). Suppose that $U G(d)$ is an optimal realization of $d$. If (i) is not satisfied, then there must exist edges $\{x, y\}$ and $\{y, z\}$ of $U G(d)$ for which

$$
d(x, z)<d(x, y)+d(y, z) .
$$

For $p, q, r \in X$, put $\delta_{p}(q, r)=\frac{1}{2}(d(p, q)+d(p, r)-d(q, r))$, and let $G$ be the weighted graph obtained from $U G(d)$ by adding a new vertex $v$, removing the edges $\{x, y\}$ and $\{y, z\}$, and adding in edges $\{x, v\},\{y, v\}$, and $\{z, v\}$ with weights $\delta_{x}(y, z), \delta_{y}(x, z)$, and $\delta_{z}(x, y)$, respectively. Then it is straightforward to check that $G$ is a realization of $d$ with $\|G\|<\|U G(d)\|$. This contradicts the optimality of $U G(d)$, and therefore (i) must hold.

Now, suppose that (ii) is not satisfied. Then there exist disjoint edges $\{x, y\}$ and $\{u, v\}$ in $U G(d)$ with

$$
d(x, y)+d(u, v)>\min \{d(x, u)+d(y, v), d(x, v)+d(y, u)\} .
$$

Assume without loss of generality that $\min \{d(x, u)+d(y, v), d(x, v)+d(y, u)\}=$ $d(x, u)+d(y, v)$. Let $G$ be the weighted graph obtained from $U G(d)$ by adding two new vertices $s, t$, removing the edges $\{x, y\}$ and $\{u, v\}$, and adding five new edges $\{x, s\}$, $\{s, u\},\{v, t\},\{t, y\}$, and $\{s, t\}$ with weights $\delta_{x}(u, y), \delta_{u}(x, y), \delta_{v}(u, y), \delta_{y}(u, v)$ and $\frac{1}{2}(d(x, y)+d(u, v)-(d(x, u)+d(y, v)))$, respectively (see Fig. 2). Again, it is easily verified that $G$ is a realization of $d$ with $\|G\|<\|U G(d)\|$, a contradiction. Thus (ii) must hold.

Now suppose that $U G(d)$ satisfies (i) and (ii), and let $G$ be any realization of $d$. We will show that for any pair $\gamma, \gamma^{\prime}$ of geodesics in $G$ joining a pair of elements $x, y \in X$
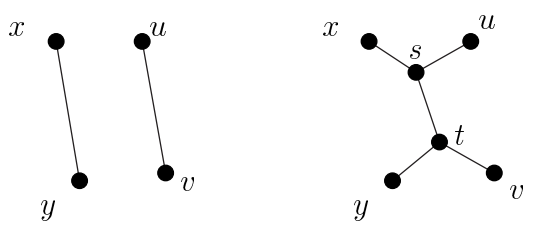

Fig. 2. The construction of a smaller realization in the proof of Theorem 1. 
with $\{x, y\}$ an edge of $U G(d)$, either $\gamma$ and $\gamma^{\prime}$ do not intersect at all, or they intersect in a single vertex. This in turn implies that $\|G\| \geq\|U G(d)\|$, and hence that $U G(d)$ is an optimal realization of $d$.

First, suppose that $\{x, y\}$ and $\{y, z\}$ are two edges in $U G(d)$ with $x, z$ distinct. Then, by Lemma 1 , any geodesics in $G$ between $x, y$ and $y, z$ must intersect only at the vertex $y$.

Next consider two edges $\{x, y\}$ and $\{u, v\}$ in $U G(d)$ with no common endpoints. If $\gamma_{x y}$ and $\gamma_{u v}$ are geodesics in $G$ with endpoints $x, y$ and $u, v$, respectively, then, by Lemma 2, $\gamma_{x y}$ and $\gamma_{u v}$ must be disjoint unless equality holds in (ii). Moreover, in the latter case, if there is some vertex $w$ in $G$ which is on $\gamma_{x y}$ and $\gamma_{u v}$, then by the triangle inequality we have $d_{G}(x, w)+d_{G}(w, u) \geq d(x, u)$ and $d_{G}(v, w)+d_{G}(w, y) \geq d(v, y)$, while similarly $d_{G}(x, w)+d_{G}(w, v) \geq d(x, v)$ and $d_{G}(u, w)+d_{G}(w, y) \geq d(u, y)$. Thus since equality holds in (ii), we see that $w$ must be the only vertex that $\gamma_{x y}$ and $\gamma_{u v}$ have in common.

\section{An Infinite Family of Graph Metrics}

We begin this section by recalling that, for $m \geq 1$ an integer, a Hadamard graph of valency $2 m$ is a connected, $2 m$-regular, bipartite, antipodal ${ }^{1}$ graph with diameter 4 . In general, it is not known for which $m$ such graphs exist. However, for our purposes it is enough to note that there exists a Hadamard graph of valency $2^{k}$ for all $k \geq 1$, since a Hadamard graph with valency $2 m$ exists if and only if a Hadamard matrix of valency $2 m$ exists, and it is known that Hadamard matrices of valency $2^{k}$ exist for all $k \geq 1$ (see Section 1.8 of [3], where more details concerning Hadamard graphs may also be found).

Now, given a Hadamard graph $H_{k}$ with valency $2^{k}, k \geq 1$, let $X_{k}$ denote its vertex set (which has cardinality $2^{k+2}$ ) and $E_{k}$ its edge set. Define a metric $d_{k}$ on $X_{k}$ as follows. Fix a vertex $x \in X_{k}$, and let

$$
Y_{k}=\left\{y: d_{H_{k}}(x, y) \in\{0,2,4\}\right\} .
$$

Note that for all $y \in Y_{k}$ we have $\bar{y} \in Y_{k}$, and that $\left|Y_{k}\right|=2^{k+1}$. Put

$$
d_{k}(x, y)= \begin{cases}2 & \text { if } x \in Y_{k} \text { and } y=\bar{x}, \\ d_{H_{k}}(u, v) & \text { else. }\end{cases}
$$

We now describe some optimal realizations of $d_{k}, k \geq 1$. Call a partition $\mathcal{A}$ of $Y_{k}$ balanced if, for each part $A \in \mathcal{A}, y \in A$ implies $\bar{y} \in A$. For each balanced partition $\mathcal{A}$ of $Y_{k}$, let $G_{k}(\mathcal{A})$ be the graph with vertex set

$$
V_{k}=X_{k} \cup\{A: A \in \mathcal{A} \text { and }|A| \geq 4\},
$$

edge set

$$
E_{k} \cup\{\{A, y\}:|A| \geq 4 \text { and } y \in A\} \cup\{\{y, \bar{y}\} \mid\{y, \bar{y}\} \in \mathcal{A}\}
$$

\footnotetext{
${ }^{1}$ Recall that a graph $G=(V, E)$ is antipodal if for every vertex $v$ in $V$ there exists a vertex $\bar{v}$ in $V$ so that for all $u \in V$ the equality $d_{G}(v, u)+d_{G}(u, \bar{v})=d_{G}(v, \bar{v})$ holds. In particular, $d_{G}(v, \bar{v})$ equals the diameter of $G$, and $\bar{v}$ is the unique vertex in $G$ that is at this distance from $v$.
} 
and edge weighting given by assigning every edge weight 1 except for those edges of the form $\{y, \bar{y}\}$, which are assigned weight 2 . So, in other words, the set $Y_{k}$ is partitioned into subsets of even cardinality; any subset with precisely two elements (necessarily a vertex and its antipode) corresponds to an edge of weight 2 , while a set with more than two elements corresponds to a star tree with edge weights all 1 , having a path of length 2 between any two of its leaves. It is straightforward to verify that $G_{k}(\mathcal{A})$ is a realization of $d_{k}$. Moreover, if $\mathcal{A}^{*}$ is the partition of $Y_{k}$ for which $|A|=2$ for all $A \in \mathcal{A}^{*}$, then $G_{k}\left(\mathcal{A}^{*}\right)=U G\left(d_{k}\right)$.

Theorem 2. Suppose $k \geq 1$, that $d_{k}$ is the metric on $X_{k}$, and that $\mathcal{A}$ is a balanced partition of $Y_{k}$. Then $G_{k}(\mathcal{A})$ is an optimal realization of $d_{k}$.

Proof. Using Theorem 1, it is straightforward to check that $U G\left(d_{k}\right)$ is an optimal realization of $d_{k}$. Moreover, since $G_{k}(\mathcal{A})$ is a realization of $d_{k}$, and $\left\|G_{k}(\mathcal{A})\right\|=\left\|U G\left(d_{k}\right)\right\|$, it immediately follows that $G_{k}(\mathcal{A})$ is an optimal realization.

Note that if $\mathcal{A}$ and $\mathcal{A}^{\prime}$ are two balanced partitions of $Y_{k}$ for which the sequences obtained by arranging the cardinalities of their parts in increasing order are distinct, then the degree sequence of $G_{k}(\mathcal{A})$ is clearly distinct from that of $G_{k}\left(\mathcal{A}^{\prime}\right)$. As a consequence of this observation and the last theorem we thus obtain the following result.

Corollary 1. Suppose $k \geq 1$. Then there are at least $p\left(2^{k-1}\right)$ optimal realizations of $d_{k}$ having distinct degree sequences, where $p\left(2^{k-1}\right)$ denotes the number of partitions of $2^{k-1}$. In particular, the number of optimal realizations with different degree sequences grows at least as quickly as $p\left(2^{k-1}\right.$ ) (a function that tends to $\left(1 / 2^{k+1} \sqrt{3}\right) e^{\sqrt{2^{k} / 3}}$ as $k \rightarrow \infty)$.

\section{Counter-examples}

We begin this section by showing that the metric $d_{1}$ provides a negative answer to Althöfer's question concerning h-optimal realizations that was given in the Introduction. The underlying graph of $d_{1}$, which is an optimal realization of $d_{1}$ by Theorem 2 , is pictured in Fig. 3(a). Note that the weight function on $U G\left(d_{1}\right)$ is the only one that can be defined on this graph to make it an an optimal realization.

Now, it is straightforward to check that the h-optimal realization $\Gamma_{d_{1}}$ of $d_{1}$ is the graph pictured in Fig. 3(b). However, clearly the graph in Fig. 3(a) cannot be obtained by deleting edges from the graph in Fig. 3(b), thus settling Althöfer's question.

We now consider Dress's conjecture concerning the injectivity of proper maps. First we identify a specific element $f_{k}$ in $T\left(d_{k}\right), k \geq 1$.

Lemma 4. For $k \geq 1$, let $f_{k}: X_{k} \rightarrow \mathbb{R}$ be defined by

$$
f_{k}(x)= \begin{cases}1 & \text { if } x \in Y_{k}, \\ 2 & \text { else }\end{cases}
$$




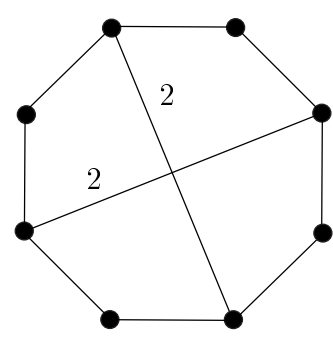

(a)

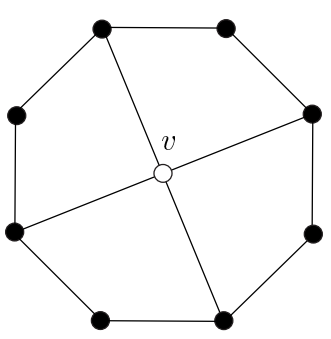

(b)

Fig. 3. Two weighted graphs, in which all unlabeled edges are assigned weight 1 . The graph in (a) is the UG-graph of $d_{1}$, which is an optimal realization of $d_{1}$ by Theorem 1, and the graph in (b) is the h-optimal realization $\Gamma_{d_{1}}$ of $d_{1}$.

Then $f_{k} \in T\left(d_{k}\right)$. Moreover, if $g \in T\left(d_{k}\right)$ with $g(y)=g(\bar{y})=1$ for some $y \in Y_{k}$, then $g=f_{k}$.

Proof. It is easily verified that for any pair of elements $x, y \in X$, we have $f_{k}(x)+$ $f_{k}(y) \geq d_{k}(x, y)$, and that for any $x \in X$ there exists some $y \in X$ such that $f_{k}(x)+$ $f_{k}(y)=d_{k}(x, y)$. It immediately follows that $f_{k} \in T\left(d_{k}\right)$.

Now suppose $y \in Y_{k}$ and $z \in Y_{k} \backslash\{y, \bar{y}\}$. Then $d_{k}(y, z)=2$, and hence $g(z) \geq$ $2-g(y)=1$. If $z \in X_{k} \backslash Y_{k}$, then either $d_{k}(y, z)=3$ or $d_{k}(\bar{y}, z)=3$, and hence $g(z) \geq 3-g(y)=2$ or $g(z) \geq 3-g(\bar{y})=2$, respectively. Thus $g(z) \geq f_{k}(z)$ for all $z \in X_{k}$, and so we must have $g=f$, since both $g$ and $f$ are elements of $T\left(d_{k}\right)$.

We now show that for all $k \geq 2$, there is an optimal realization of $d_{k}$ that provides a counter-example to Dress's conjecture.

Theorem 3. Suppose $k \geq 2$, and that $\mathcal{A}$ is a balanced partition of $Y_{k}$. If $\psi: V_{k} \rightarrow T\left(d_{k}\right)$ is a proper map, then $\psi(A)=f_{k}$ for all $A \in \mathcal{A}$ with $A \in V_{k}$. In particular, if $\mathcal{A}$ contains at least two parts having cardinality at least 4 , then the map $\psi$ is not injective.

Proof. Let $\mathcal{A}$ be a balanced partition of $Y_{k}$ containing at least one part $A$ with $|A| \geq 4$. Put $G_{k}(\mathcal{A})=\left(V_{k}, E, w\right)$.

Now, suppose $\psi: V_{k} \rightarrow T\left(d_{k}\right)$ is a proper map. Let $A \in \mathcal{A}$ with $|A| \geq 4$, and put $g_{A}=\psi(A)$, so that in particular $g_{A} \in T\left(d_{k}\right)$. In case $y \in A$, then by properties (I) and (II) of proper maps,

$$
g_{A}(y)=d_{\infty}\left(g_{A}, h_{y}\right)=d_{\infty}(\psi(A), \psi(y))=w(\{A, y\})=1 .
$$

Moreover, since $\bar{y}$ is also in $A$, the same argument gives $g_{A}(\bar{y})=1$. Hence, by Lemma 4, $g_{A}=f_{k}$.

As an illustration of this theorem, in Fig. 4 we present an optimal realization of $d_{2}$ in which both of the white auxilliary vertices $A_{1}$ and $A_{2}$ in $\mathcal{A}$ are mapped by any proper map $\psi: V_{2} \rightarrow T\left(d_{2}\right)$ to the element $f_{2}$ in $T\left(d_{2}\right)$. 


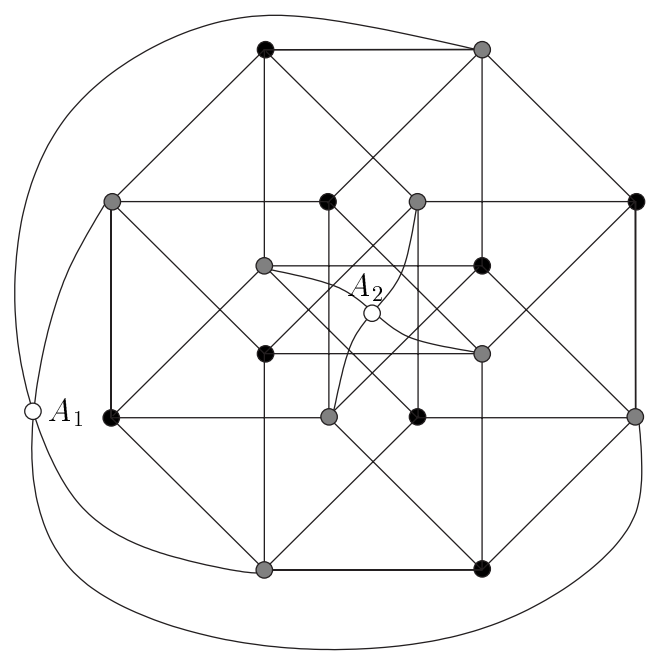

Fig. 4. An optimal realization $G_{2}(\mathcal{A})$ of the metric $d_{2}$ corresponding to a partition $\mathcal{A}$ of $Y_{2}$ into two parts with cardinality 4 . The vertices in the set $Y_{2}$ are shown in gray, and the two vertices $A_{1}$ and $A_{2}$ corresponding to the elements in $\mathcal{A}$ in white. All edges have weight 1.

In conclusion, for any metric $d$, it might still be of interest to see whether at least one optimal realization of $d$ can always be obtained by deleting some edges from the hoptimal realization $\Gamma_{d}$. In regards to this, it is worth noting that it can be shown that there always exists an optimal realization $G=(V, E, w)$ of $d$, with the property that all proper maps $\psi: V \rightarrow T(d)$ are injective. In future work we will investigate the consequences of this fact for obtaining a better understanding of the relationship between optimal and h-optimal realizations.

\section{Acknowledgements}

The authors thank an anonymous referee for a number of helpful comments which have improved the clarity of the presentation.

\section{References}

1. I. Althöfer, On optimal realizations of finite metric spaces by graphs, Discrete Comput. Geom. 3 (1988), $103-122$.

2. H. -J. Bandelt and A. Dress, A canonical decomposition theory for metrics on a finite set, Adv. Math. 92 (1992), 47-105.

3. A. E. Brouwer, A. M. Cohen, and A. Neumaier, Distance-Regular Graphs, Springer-Verlag, Berlin, 1989.

4. M. Chrobak and L. Larmore, Generosity helps or an 11-competitive algorithm for three servers, J. Algorithms 16 (1994), 234-263.

5. A. Dress, Trees, tight extensions of metric spaces, and the cohomological dimension of certain groups: a note on combinatorial properties of metric spaces, Adv. Math. $\mathbf{5 3}$ (1984), 321-402.

6. A. Dress, K. T. Huber, A. Lesser, and V. Moulton, Hereditarily optimal realizations of consistent metrics, Ann. Comb. 10 (2006), 63-76. 
7. A. Dress, K. T. Huber, and V. Moulton, An explicit computation of the injective hull of certain finite metric spaces in terms of their associated Buneman complex, Adv. Math. 168 (2002), 1-28.

8. A. Dress, K. T. Huber, and V. Moulton, Hereditarily optimal realizations of metrics: Why are they relevant in phylogenetic analysis and how does one compute them?, in Algebraic Combinatorics and Applications, pp. 110-117, Springer-Verlag, New York, 2001.

9. A. Dress, V. Moulton, and W. Terhalle, T-theory: an overview, European J. Combin. 17 (1996), 161-175.

10. W. Imrich, J. Simoes-Pereira, and C. Zamfirescu, On optimal embeddings of metrics in graphs, J. Combin. Theory B 36(1) (1984), 1-15.

11. J. Koolen, V. Moulton, and U. Tönges, A classification of the six-point prime metrics, European J. Combin. 21 (2000), 815-829.

12. J. M. S. Simões-Pereira, A note on optimal and suboptimal digraph realizations of quasidistance matrices, SIAM J. Algebraic Discrete Methods 5 (1984), 117-132.

13. P. Winkler, The complexity of metric realization, SIAM J. Discrete Math. 1 (1988), 552-559.

Received November 22, 2006, and in revised form March 17, 2007. Online publication August 24, 2007. 